\title{
DETECÇÃO DE FORMAÇÃO EROSIVA (VOÇOROCA) POR MEIO DE CLASSIFICAÇÃO HIERÁRQUICA E POR ÁRVORE DE DECISÃo
}

\author{
Gully erosion detection by hierarchical classification and tree decision
}

\author{
ANDREA TEDESCO \\ ALZIR FELIPPE BUFFARA ANTUNES \\ LUIZ OCTÁVIO OLIANI
}

Universidade Federal do Paraná

Programa de Pós-Graduação em Ciências Geodésicas

Centro Politécnico - Jardim das Américas

81.531-990 - Curitiba - PR - Brasil

atedesco@uepg.br; felipe@ufpr.br; luizoliani91@gmail.com

\section{RESUMO}

Este estudo teve por objetivo verificar a possibilidade de reconhecimento de feições erosivas do tipo voçoroca utilizando análise orientada a objeto (OBIA - ObjectBased Image Analysis). A área de estudo está localizada no município de Uberlândia, em Minas Gerais. Em função do objetivo, definiu-se uma rede semântica hierárquica multinível para representação do conhecimento especialista. Foram usados dados espectrais oriundos de imagem IKONOS e dados de intensidade e altimétricos provenientes de perfilamento com ALS (Airborne Laser Scanner). Os objetos foram gerados por meio de segmentação multirresolução (FNEA-Fractal Net Evolution Approach) aplicada aos dados espectrais e altimétricos. O reconhecimento das feições foi realizado por classificação hierárquica e por árvores de decisão (algoritmo CART-Classification And Regression Trees). A metodologia permitiu a identificação da relevância dos dados de entrada, parâmetros de segmentação e atributos a serem usados para classificação das voçorocas. Os resultados obtidos com a classificação hierárquica e com CART foram bastante similares, os quais evidenciaram a possibilidade do uso do método semiautomatizado a partir dos parâmetros previamente identificados e analisados.

Palavras-chave: Análise Orientada a Objeto; Classificação Hierárquica; Árvores de Decisão; Lógica Fuzzy; Mapeamento de Voçorocas. 


\section{ABSTRACT}

This research aims at verifying the possibility to recognize gully erosion using Object-Based Image Analysis (OBIA). The studied area is located in Uberlândia, Minas Gerais. In according to research purpose, a multiscale hierarchical semantic net was defined to represent the expertise knowledge. Spectral data from IKONOS imagery, and intensity and altimetric data from ALS (Airborne Laser Scanner) were used. The objects were generated by multirresolution segmentation (FNEA-Fractal Net Evolution Approach) applied to spectral and altimetric data. The feature recognition was performed by hierarchical classification and tree decision algorithm (CART - Classification and Regression Trees). By means of proposed methodology, it was possible to identify the relevant input data, segmentation parameters and attributes to classify gully erosion. The results were similar; either by hierarchical classification or by CART, in which the possibility to use semiautomated methods from a set of previously identified and analyzed parameters was shown.

Keywords: Object-Based Image Analysis; Hierarchical Classification; Decision Tree; Fuzzy Logic; Gully Erosion Mapping.

\section{INTRODUÇÃO}

As voçorocas são processos erosivos caracterizados por depressões profundas, circundadas por vertentes quase verticais, que se alargam nas proximidades das cabeceiras devido à intensa atividade erosiva regressiva e se afunilam junto à foz do curso-d'água que a percorre, cortando sedimentos arenosos de fraca coesão, sem apresentar forte declive longitudinal (VIEIRA, 1978). Ocasionam a perda de solos e constituem os processos erosivos de maiores proporções e, consequentemente, de maiores prejuízos financeiros, sociais e ambientais. Para que ações mitigatórias ou corretivas possam ser aplicadas, faz-se necessário o mapeamento e monitoramento dessas feições erosivas, os quais podem ser realizados por medidas conduzidas no local ou por sensoriamento remoto.

As medidas efetuadas no local, apesar de amplamente utilizadas, requerem o caminhamento e instalação de equipamentos nas bordas e interior das voçorocas, o que pode acarretar o agravamento dos processos erosivos já instalados e o risco para os executores do levantamento. Em função disso, o monitoramento por sensoriamento remoto torna-se bastante atrativo.

Entre as características das voçorocas (identificadas em imagens orbitais), que as diferem das demais feições urbanas e rurais, estão: a heterogeneidade espectral (mistura de solos, vegetação e água), a variabilidade espacial (formato irregular, dimensões variadas, assimetria e relação largura/comprimento variável) e a variação altimétrica (de centímetros a metros, com declividade bastante acentuada nas bordas).

Na condução de estudos de voçorocas por sensoriamento remoto, devido à heterogeneidade espectral, o uso apenas de informações espectrais é insuficiente 
para discriminar a classe voçoroca das demais. Isso sugere que a incorporação de informações auxiliares, como as altimétricas, geométricas e de contexto, possa auxiliar na discriminação dessa classe.

Devido às especificidades da classe voçoroca, bem como à dimensionalidade e variabilidade dos dados espectrais e auxiliares, as técnicas de mineração de dados (como os algoritmos de árvore de decisão) apresentam-se como alternativa para o processamento.

Nesse contexto, esse estudo teve por objetivo definir uma metodologia semiautomatizada para o mapeamento da classe de uso do solo "voçoroca", com base em abordagem orientada a objeto, incorporando informações altimétricas e espectrais no processamento. Para tal, foram utilizados os métodos de classificação hierárquica e classificação por árvores de decisão. Como dados de entrada foram utilizados informações espectrais, oriundas de imagem IKONOS, e dados altimétricos e de intensidade, oriundos de ALS. O estudo foi conduzido para uma área localizada no município de Uberlândia/MG, Brasil, selecionada em função da existência de voçoroca no local e da disponibilidade dos dados.

\section{MAPEAMENTO E MONITORAMENTO DE VOÇOROCAS}

$\mathrm{O}$ mapeamento e monitoramento de áreas atingidas por voçorocas, comumente, é realizado a partir de medidas efetuadas no local, com a técnica de estaqueamento (HESSEL e VAN ASCH, 2003; GUERRA, 2005; MORGAN, 2005). Apesar deste método ainda ser amplamente utilizado, outras formas de levantamentos de dados têm sido aplicadas, fornecendo maior amostragem de dados, rapidez e precisão, como é o caso dos levantamentos topográficos convencionais ou com uso de equipamento laser (Light Amplification by Stimulated Emission of Radiation) escâner terrestre (PERROY et al., 2010).

Alternativamente aos levantamentos no local, técnicas aerofotogramétricas podem ser usadas para geração de Modelo Digital de Terreno (MDT) e fotointerpretação, com a finalidade de mapear voçorocas (MARTÍNEZCASASNOVAS, 2003; DABA, RIEGER e STRAUSS, 2003; MARTÍNEZCASASNOVAS, RAMOS e POESEN, 2004). O uso de aerofotos digitais e de alta resolução tem permitido estudos mais detalhados sobre as voçorocas (MARZOLFF e POESEN, 2009).

Imagens obtidas por sensores a bordo de satélites permitem a identificação de áreas com risco de erosão (KING et al., 2005; VRIELING, RODRIGUES e STERK, 2005). Com auxílio de MDT, gerado a partir de dados altimétricos de varredura a laser(ALS), pode-se realizar o cálculo volumétrico e estimar perdas de solo (JAMES, WATSON e HANSE, 2007; EUSTACE, PRINGLE e WITTE, 2009).

Atualmente, os estudos mostram uma busca por métodos automáticos ou semiautomáticos, em abordagem orientada a objeto, para detecção, mapeamento e monitoramento, bem como para cálculos volumétricos e modelos preditivos de risco de erosão (PERROY et al., 2010; SHRUTHI, KERLE e JETTEN, 2011 e 2012; JOHANSEN et al., 2012; WANG et al., 2014). Alkhasawneh et al. (2014) 
realizaram estudo para determinar a susceptibilidade a deslizamentos e movimentos de massa, em função de variáveis topográficas e geológicas, com auxílio de algoritmos de árvores de decisão.

\section{3. ÁREA DE ESTUDO}

A área de estudo está localizada na zona rural do município de Uberlândia/MG, na Fazenda Experimental do Glória, pertencente à Universidade Federal de Uberlândia. A região pertence ao bioma Cerrado, apresentando áreas de relevo dissecado (nas quais os processos erosivos são bastante intensos), com clima temperado - Cwa (estações de verão e inverno bem definidas) e média anual de precipitação de $1.500 \mathrm{~mm}$ por ano (LEAL E RODRIGUES, 2011).

A figura 1 ilustra a localização da área de estudo e algumas características da voçoroca (por meio de registros fotográficos). A presença de solo, vegetação e água dificulta a classificação espectral dessa feição, devido à mistura de elementos constituintes (foto 1). A variação altimétrica, mostrada na foto 2 , pode auxiliar no processo de classificação. As direções das tomadas das fotos são indicadas em amarelo (foto 1 - cabeceira da voçoroca) e vermelho (foto 2 - canal principal da voçoroca).

Figura 1 - Localização e registros fotográficos da área de estudo.

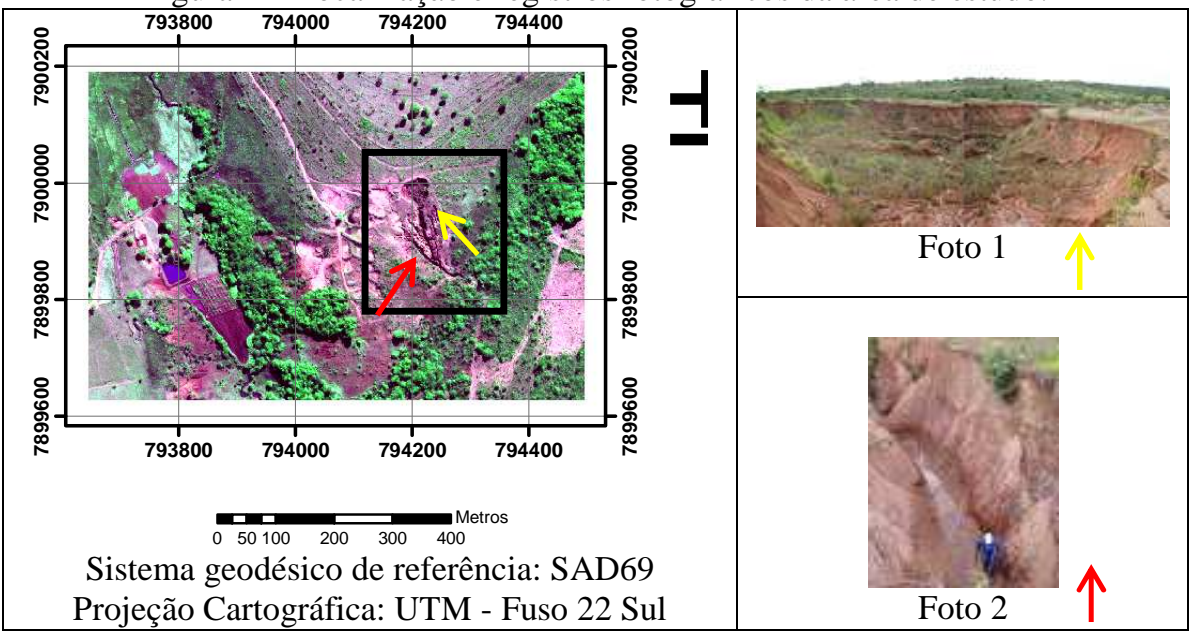

\section{FUNDAMENTAÇÃO TEÓRICA}

\subsection{Segmentação de Imagens}

A segmentação é a divisão de uma imagem digital em várias regiões, gerando objetos que apresentem alguma uniformidade interna em relação a uma propriedade 
da imagem, e contrastem em relação à sua vizinhança (GONZALES e WOODS, 2008).

Baseados na natureza fractal das imagens de sensoriamento remoto, Baatz e Schäpe (1999) introduziram a FNEA (Fractal Net Evolution Approach). A técnica consiste em extrair os objetos pela segmentação e, posteriormente, classificá-los por meio da lógica fuzzy. A segmentação é feita de forma fina e grosseira, simultaneamente, originando escalas de segmentação (procedimento também denominado de segmentação multirresolução). Cada escala gerará um nível de objetos e a conexão dos níveis ocorre de forma hierárquica. Os sub objetos (gerados nas escalas menores) estão contidos nos super objetos (gerados em escalas maiores, a partir da junção dos sub objetos).

$\mathrm{Na}$ rede hierárquica, cada objeto conhece seus vizinhos adjacentes, seus sub objetos e seus super objetos, o que permite o uso de informação contextual na classificação, por meio de operações de vizinhança entre os objetos em rede. A informação espectral pode ser combinada com a forma dos objetos e características de textura para melhorar a classificação (BAATZ e SCHÄPE, 1999).

No início do processo, cada pixel da imagem é considerado como sendo um objeto. A cada passo, os dois objetos que geram um novo objeto mais uniforme, em termos espectrais e espaciais, são fundidos (cada objeto e seus vizinhos são analisados). O par de vizinhos que mais se assemelham é escolhido para a fusão. Caso exista mais de um vizinho semelhante, é escolhido o que possui menor fator de heterogeneidade, composto pelas heterogeneidades espectral e de forma. O processo continua até que um número de iterações seja atingido ou não existam mais fusões.

A heterogeneidade espectral $\left(h_{\text {espectral }}\right)$ é descrita pela variância ou desvio padrão dos pixels (do segmento) em cada banda. É adotado o somatório dos desvios padrão $(\sigma)$ dos valores espectrais em cada banda (c) ponderados com o peso $(\mathrm{w})$ atribuído para cada banda (TRIMBLE, 2012):

$$
h_{\text {espectral }}=\sum_{c} w_{c} \cdot \sigma_{c}
$$

A heterogeneidade de forma $\left(h_{\text {forma }}\right)$ é função dos coeficientes de compacidade e suavidade, e é dada por (TRIMBLE, 2012):

$$
h_{\text {forma }}=w_{\text {compac }} \cdot h_{\text {compac }}+\left(1-w_{\text {compac }}\right) \cdot h_{\text {suav }}
$$

A compacidade representa o quão compactos estão os pixels de um determinado objeto e a suavidade representa o grau de irregularidade do contorno de um objeto. O coeficiente de compacidade $\left(h_{\text {compac }}\right)$ é calculado comparando o perímetro do objeto com o perímetro da circunferência equivalente. O coeficiente de suavidade $\left(h_{\text {suav }}\right)$ é obtido pela comparação do perímetro da região com o perímetro do menor retângulo envolvente. O peso para o critério de compacidade 
assume valores no intervalo de 0 a 1 . O custo de fusão de dois objetos (f) é dado por (TRIMBLE, 2012):

$$
f=w \cdot h_{\text {espectral }}+(1-w) \cdot h_{\text {forma }}
$$

sendo:

$\mathrm{w}, \mathrm{o}$ peso atribuído à cor $(0 \leq \mathrm{w} \leq 1)$;

$h_{\text {espectral }}$, a heterogeneidade espectral;

$h_{\text {forma }}$, a heterogeneidade espacial (da forma).

Haverá fusão entre os objetos se o custo de fusão for menor que um critério definido como escala. $\mathrm{O}$ fator de escala (FE) e os pesos para a informação espectral e de forma (bem como o peso para a compacidade do parâmetro de forma) devem ser definidos pelo usuário.

\subsection{Classificação de Imagens}

A classificação de imagens consiste em separar conjuntos de pixels que apresentem características similares (por exemplo, espectral, de forma ou textura) em classes de uso ou cobertura do solo. O resultado da classificação é um mapa temático mostrando a distribuição geográfica das classes.

\subsubsection{Classificação Baseada em Relações de Hierarquia}

A classificação hierárquica, integrante da FNEA, baseia-se em lógica fuzzy e é realizada com base nos diferentes níveis provenientes da segmentação multirresolução. As feições são classificadas de acordo com o nível em que melhor forem segmentadas. A classificação é realizada com base na rede hierárquica estabelecida, nas amostras selecionadas para treinamento dos algoritmos e nos atributos selecionados como descritores para cada classe (BAATZ e SCHÄPE, 1999).

\subsubsection{Classificação Por árvores de Decisão}

As árvores de decisão são uma representação gráfica para um procedimento de classificação ou de regressão, sendo um método de aprendizado supervisionado. Elas possibilitam a extração de conhecimento a partir de exemplos de entrada, na forma de amostras de treinamento. Os dados originais são reduzidos a uma árvore de predição que representa um conjunto de regras de decisão. Sua representação simplificada assume a forma de uma árvore invertida. A árvore apresenta uma divisão hierárquica para a rotulagem dos objetos, permitindo que sejam analisadas quais as características mais relevantes na separação das classes (TSO e MATHER, 2009).

O objetivo principal dos algoritmos de árvore de decisão é encontrar a menor árvore de decisão possível, coerente com os exemplos de treinamento, atingindo-se uma classificação correta com um número pequeno de testes. A tarefa é testar o 
atributo de maior importância em primeiro lugar, ou seja, o atributo que influencia predominantemente a classificação de determinado exemplo.

O algoritmo CART, proposto por Breiman, Friedman, Olsen e Stone (1984), permite a formação de apenas dois ramos (dois filhos) em cada etapa do processo, ou seja, a árvore de decisão gerada será sempre uma árvore binária (TSO e MATHER, 2009).

\subsubsection{Avaliação da Classificação - Matriz de Confusão}

A classificação, por ser uma generalização das informações constantes em uma cena, necessita de uma avaliação para expressar o quão confiável é o mapa temático dela derivado. A avaliação pode ser realizada comparando-se o resultado com um mapa de referência, o qual se assume como correspondente à verdadeira classificação, ou com dados levantados no local da cena, referentes à verdade de campo. Quando se usa o método supervisionado, pode-se também realizar a avaliação da classificação em função dos dados de treinamento (amostras), ou destes em conjunto com dados de teste (validação cruzada).

A matriz de confusão é uma maneira de representação da avaliação da classificação e mostra os conflitos que existem entre as classes. Quando a avaliação é realizada em função dos dados de treinamento, as linhas correspondem às amostras selecionadas (assumidas como referência) e as colunas correspondem aos dados classificados. Cada célula da matriz apresenta o valor do pixel que deveria ser classificado como a classe especificada na linha e que foi classificado segundo a classe especificada na coluna. Para uma classificação perfeita, a matriz apresenta somente os valores da diagonal principal, o que dificilmente ocorre. Os pixels classificados incorretamente são os que se encontram fora da diagonal principal. A partir da matriz de confusão, pode-se calcular a qualidade global (Q) da classificação (CENTENO, 2009; TSO e MATHER, 2009):

$$
Q=\frac{N C}{N T}
$$

sendo NC o número de pontos corretamente classificados (soma dos valores da diagonal principal) e NT o número total de pontos.

Para obter a acurácia de cada classe separadamente, os conceitos de acurácia do produtor e do usuário podem ser usados. A acurácia do produtor está relacionada com os erros de omissão (EOM), que correspondem aos pixels pertencentes a uma determinada classe que foram erroneamente classificados como sendo de outra, ou seja, foram omitidos da classe assumida como verdadeira (CENTENO, 2009; TSO e MATHER, 2009):

$$
E O M=\frac{S L(i)-M(i i)}{S L i}
$$


sendo SL(i) a soma parcial da linha i, e M(i,i) o elemento da diagonal principal na linha i.

A acurácia do usuário está relacionada com os erros de inclusão (EIN), que correspondem aos pixels que foram incluídos na classificação e que não correspondem aos que foram assinalados como verdadeiros (CENTENO, 2009; TSO e MATHER, 2009):

$$
E I N=\frac{S C(i)-M(i i)}{S C i}
$$

sendo SC(i) a soma parcial da coluna i.

Outra forma de avaliar a acurácia da classificação é através do índice Kappa, ou coeficiente de concordância, proposto por Cohen em 1960, expresso por (TSO e MATHER, 2009):

$$
k=\frac{N * \sum_{i=1}^{M} M(i, i)-\sum_{i=1}^{M} S L(i) * S C(i)}{N^{2}-\sum S L(i) * S C(i)}
$$

sendo $\mathrm{N}$ o número de amostras (ou observações) e $\mathrm{M}$ o número de classes. Um valor de kappa entre 0,8 e 1,0 expressa uma classificação excelente, entre 0,6 e 0,8 muito boa, entre 0,4 e 0,6 boa, entre 0,2 e 0,4 razoável, entre 0 e 0,2 ruim e abaixo de 0 péssima (LANDIS e KOCH, 1977). Uma das vantagens para uso do kappa é que ele incorpora a informação dos pixels mal classificados, e não apenas dos bem classificados como a acurácia total (qualidade global).

\subsection{Lógica fuzzy}

A lógica fuzzy, ou teoria de conjuntos difusos ou nebulosos, proposta por Zadeh em 1965, baseia-se na teoria clássica de conjuntos. Na classificação de imagens, ela permite que as incertezas possam ser expressas por meio de funções de pertinência (o grau de pertinência assume valores no intervalo de 0 a 1 ), ao invés do uso de regras de decisão determinísticas. Usando-se regras determinísticas, um pixel pertencerá ou não a uma dada classe. Usando-se a lógica fuzzy, um pixel pode pertencer a uma classe com um grau de pertinência, e a outra classe com outro grau de pertinência. Isso permite a representação do conhecimento do intérprete humano (ANTUNES, 2003; TSO e MATHER, 2009). No programa eCognition estão implementadas as funções ilustradas na figura 2. O programa sugere a função de pertinência de acordo com a amostra de treinamento fornecida, e é possível ajustar o intervalo de valores, bem como a função. 
Figura 2 - Funções de pertinência fuzzy disponíveis no programa eCognition. Adaptado de TRIMBLE (2012)

\begin{tabular}{|c|c|c|c|}
\hline 5 & maior que & $\Delta$ & intervalo linear (triangular) \\
\hline & menor que & & intervalo linear (triangular invertida) \\
\hline & maior que (booleana) & & singleton (exatamente um valor) \\
\hline & menor que (booleana) & & gaussiana aproximada \\
\hline & maior que (linear) & & aproximadamente o intervalo \\
\hline 2 & Menor que (linear) & & intervalo total \\
\hline
\end{tabular}

\section{MATERIAIS}

Para o desenvolvimento da metodologia foram usados:

a) Dados ALS altimétricos e de intensidade: obtidos com equipamento ALTM 2025 (Airborne Laser Terrain Mapper), do fabricante Optech Inc.; voos efetuados de janeiro a junho de 2004, com altura de voo de $1.000 \mathrm{~m}$; densidade média de 1,89 pontos por $\mathrm{m}^{2}$; distância média entre pontos de $1,23 \mathrm{~m}$; qualidade planimétrica após processamento (segundo o fabricante) de $0,5 \mathrm{~m}$ a $1,0 \mathrm{~m}(1 / 1000 \times \mathrm{H}$ a $1 / 2000 \times \mathrm{H}$, sendo $\mathrm{H}$ a altura de vôo). Altitudes ortométricas, relativas ao Datum de Imbituba.

b) Imagem IKONOS multiespectral: corrigida pelos parâmetros orbitais e reamostrada por convolução cúbica. Foram disponibilizadas as bandas 1, 2, 3 e 4 (azul, verde, vermelho einfravermelho) fusionadas com a Pan (pancromática), com resolução espacial de $1 \mathrm{~m}$.

Para o processamento dos dados foram utilizados os programas: ENVI (Environment for Visualizing Images) 4.7, ALDPAT (Airborne LiDAR Data Processing and Analysis Tools), eCognition Developer 8.8, ERDAS Imagine $9.1 \mathrm{e}$ ArcGis 9.

\section{MÉTODOS}

\subsection{Pré-Processamento dos Dados}

Os dados ALS (coordenadas Leste e Norte na projeção UTM, altitude e intensidade do retorno do sinal) foram usados para gerar o MDS (Modelo Digital de Superfície) e a imagem de intensidade. Sobre os dados altimétricos realizou-se a triangulação de Delaunay e, posteriormente, a interpolação linear. Sobre os dados de intensidade, aplicou-se a interpolação linear. Os procedimentos realizados (com auxílio do programa ENVI), sobre os dados altimétricos e de intensidade, permitiram a transformação dos pontos irregularmente espaçados em uma grade regular (imagem raster), com resolução espacial de $1 \mathrm{~m}$ (compatível com a resolução espacial da imagem IKONOS e com o espaçamento dos dados ALS).

Sobre o MDS realizou-se o procedimento de filtragem dos pontos acima do terreno, com auxílio do programa ALDPAT (ZHANG \& CUI, 2007), visando a 
geração do MDT (figura 3). O filtro ATIN (Adaptive TIN Filter), desenvolvido por Axelsson (2000), apresentou a melhor definição do contorno da voçoroca.

Como o MDS apresenta a variação altimétrica das feições em função das altitudes, as alturas dos objetos não são conhecidas. No processo de geração do MDSN as variações altimétricas são normalizadas, sendo todas as alturas reduzidas a um mesmo plano (subtração do relevo) e as alturas dos objetos são acessadas diretamente. O MDSN (figura 4) foi obtido usando-se o princípio de matemática de bandas, subtraindo-se o MDT do MDS, com auxílio do programa ENVI. O MDSN foi usado na discriminação entre vegetação arbórea e rasteira. A partir do MDT gerou-se o mapa de contornos (curvas de nível) e, a partir dele, o mapa de declividade (figura 5). A declividade foi usada na discriminação da classe voçoroca, pois a borda da mesma apresenta declividade acentuada. Esses procedimentos foram realizados com auxílio dos programas ERDAS e ArcGIS.

Figura 3 - Modelo Digital de Terreno.

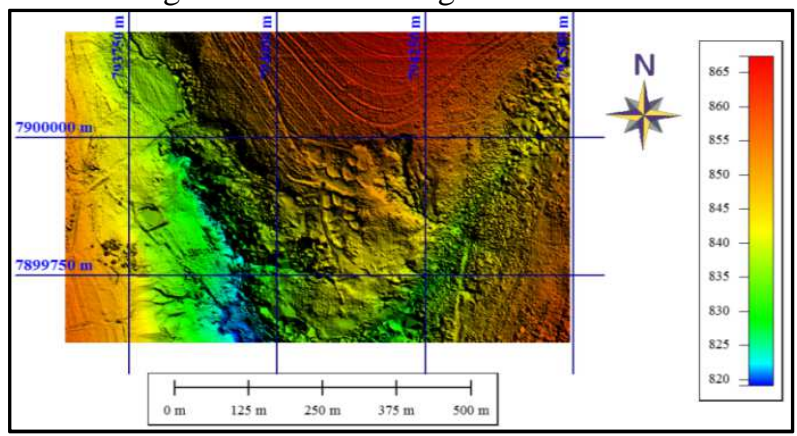

Figura 4 - Modelo Digital de Superfície Normalizado.

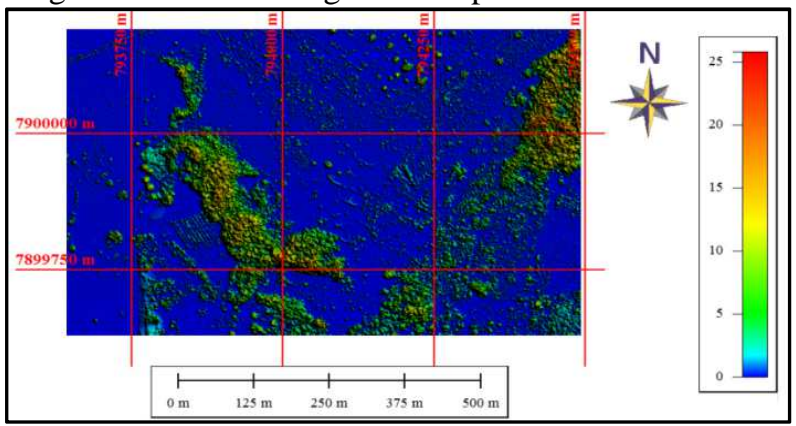

A partir das bandas espectrais relativas aos comprimentos de onda do infravermelho próximo e do vermelho (bandas 4 e 3 da imagem IKONOS, respectivamente), gerou-se o NDVI (Normalized Difference Vegetation Index) para 
melhorar a discriminação das áreas vegetadas e não vegetadas $($ NDVI $=(B 4-$ $\mathrm{B} 3) /(\mathrm{B} 4+\mathrm{B} 3))$. O procedimento foi realizado com auxílio do programa ENVI. O NDVI varia de $-1 \mathrm{a}+1 \mathrm{e}$, quanto maior esse valor, maior a presença de vegetação. Foram utilizados diretamente os valores obtidos, sem necessidade de normalização dos mesmos. A figura 6 ilustra a imagem NDVI obtida, na qual pode-se perceber a nítida discriminação da vegetação e não vegetação.

Figura 5 - Mapa de Declividade.

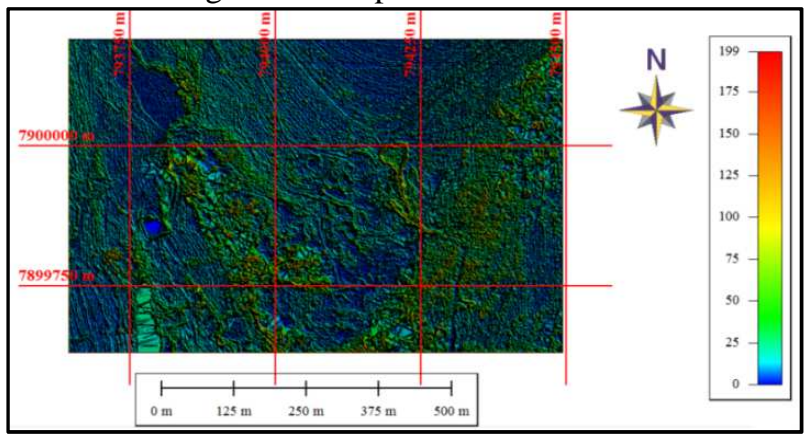

Figura 6 - Imagem NDVI.

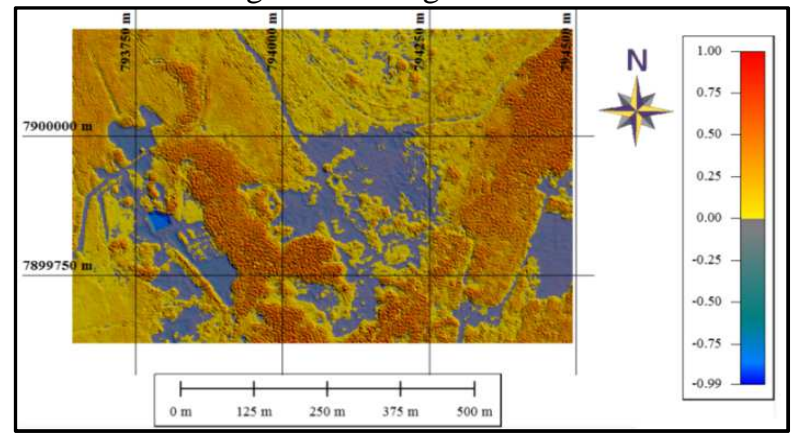

Para realizar o processamento conjunto dos dados espectrais e altimétricos há a necessidade de que ambos estejam perfeitamente registrados, por isso optou-se em realizar o registro dos dois conjuntos de dados (ALS e IKONOS) ao invés do georreferenciamento. A imagem IKONOS foi registrada usando-se a imagem de intensidade como referência. Foram usados 10 pontos de controle. Por se tratar de ambiente rural, não foi possível distribuir os pontos por toda a cena, tendo havido concentração em alguns locais, como no caso da área urbanizada. A distribuição se deu em virtude da disponibilidade de feições homólogas bem definidas em ambas as imagens e do controle do erro do registro, estipulado a priori como inferior a dois 
pixels. O erro máximo do registro em $\mathrm{X}$ (Ex) foi de $0,8653 \mathrm{~m}$ e em y (Ey) foi de $-1,3139 \mathrm{~m}$. O erro total foi de $0,8698 \mathrm{~m}$, inferior a um pixel.

\subsection{Segmentação}

A segmentação dos dados foi realizada pela técnica FNEA, implementada no programa eCognition como algoritmo de segmentação multirresolução. Foram utilizados como dados de entrada as quatro bandas da imagem IKONOS, a imagem NDVI, a imagem de intensidade do ALS, o MDT, o MDSN e o mapa de declividade. Variou-se o fator de escala (FE) de 5 a 100 (com intervalos múltiplos de 5, ex: 5-10-15-20...), e $\mathrm{FE}=125$ e $\mathrm{FE}=150$. As segmentações obtidas com os diferentes fatores de escala foram analisadas, visando identificar em que nível de segmentação as feições da voçoroca (cabeceira, dígitos e bordas) foram delineadas pelos segmentos.

Como parâmetros de entrada para o algoritmo, atribuiu-se maior peso à cor $(0,9)$ do que à forma $(0,1)$, pois, após realização de testes, verificou-se que a variação desses parâmetros não alterou as segmentações neste estágio do processamento. Quanto ao parâmetro de heterogeneidadede forma, usou-se compacidade $=0,5$ (o que acarreta na suavidade $=0,5$, conforme equação 2 ). Todos os valores foram definidos de forma empírica.

A figura 7 mostra os segmentos obtidos para $\mathrm{FE}=30$ e $\mathrm{FE}=150$. Com o $\mathrm{FE}=150$ é possível separar as áreas vegetadas e não vegetadas, bem como é possível delinear o contorno das feições maiores da voçoroca. Usando-se os segmentos mais detalhados é possível distinguir vegetação arbórea e rasteira, bem como classificar os dígitos (canais secundários e mais estreitos) da voçoroca.

Figura 7 - Segmentação com FE=30(a) e FE=150(b).

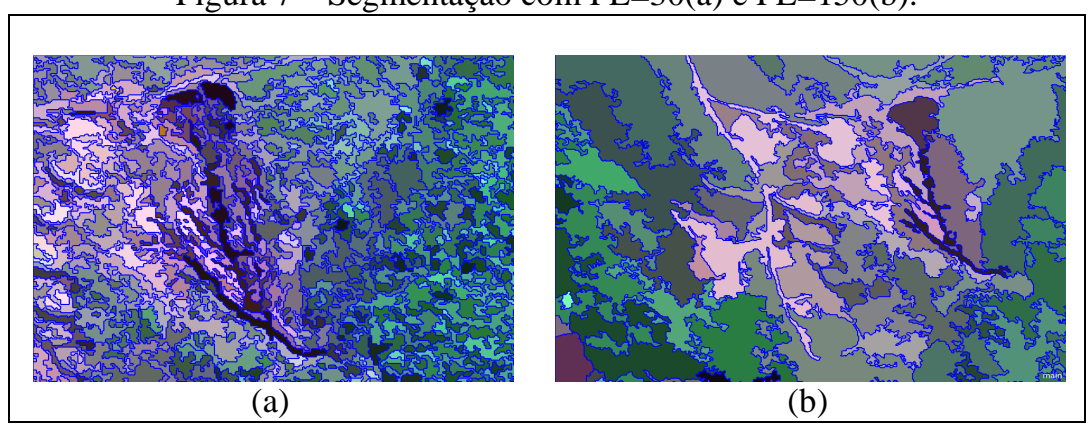

\subsection{Planejamento da Classificação}

\subsubsection{Definição das Classes e da Rede Semântica}

A chave de fotointerpretação foi o primeiro passo para a criação da rede semântica da classificação. Para este estudo, foram analisadas as características que 
poderiam ser usadas na discriminação de cada classe. O quadro 1 mostra as chaves de interpretação, para uma composição colorida $\mathrm{R}=$ banda $3, \mathrm{G}=$ banda 4 e $\mathrm{B}=$ =banda 1. Foram analisados os aspectos referentes à cor, forma, tamanho, textura e variação de altura em relação ao solo.

Por se tratar de ambiente rural, poucas classes foram identificadas para o interesse desse estudo, sendo essas: vegetação (arbórea e rasteira), corpos-d'água e solo (solo exposto e voçoroca). A definição ocorreu em função do reconhecimento de campo no local e da percepção dos autores.

Quadro 1 - Chaves de interpretação das classes de cobertura do solo para a composição colorida $\mathrm{R}=3, \mathrm{G}=4, \mathrm{~B}=1$.

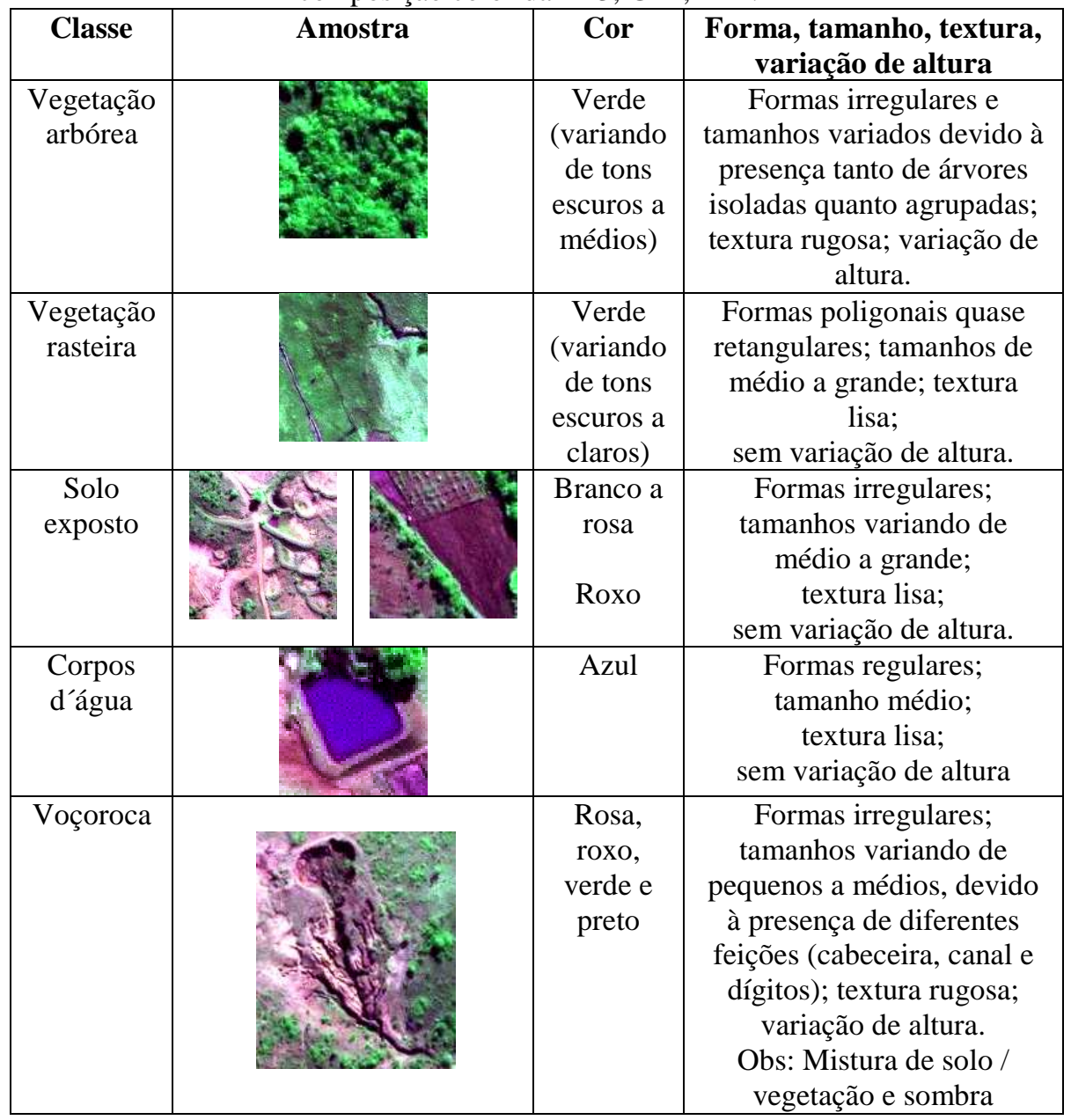


6.3.2 Definição dos Atributos

Foi realizada uma análise exploratória dos diversos atributos pré-definidos no programa eCognition. Neste estudo, são apresentados os resultados obtidos usando apenas os atributos de cor e, por isso, na segmentação usou-se peso maior nessa característica. Há que se ressaltar que este programa utiliza a variação de altura do MDSN e dos valores de declividade como um valor digital do pixel, ou seja, como uma variação de cor.

\subsubsection{Descrição das Classes}

A partir das chaves de interpretação e da análise dos atributos, foram definidos os descritores de cada classe, bem como as funções de pertinência fuzzy, a serem usados na classificação hierárquica. Esses descritores foram definidos de forma empírica, baseados no conhecimento dos autores e na avaliação dos resultados obtidos quando do uso de diferentes descritores. Para algumas classes usou-se apenas o algoritmo classificador do vizinho mais próximo (NN - nearest neighbour), e para outras classes foram usadas também funções de pertinência fuzzy. As funções de pertinência foram sugeridas pelo programa, a partir das amostras de treinamento fornecidas, e suas formas e intervalos de valores foram adaptados de acordo com o conhecimento dos autores. A figura 8 ilustra as funções fuzzy utilizadas, o intervalo de valores, classe para a qual foi utilizada e banda à qual foi aplicada.

Figura 8 - Funções de pertinência fuzzy utilizadas.

\begin{tabular}{|c|c|c|}
\hline Água - Infravermelho & $\begin{array}{c}0.033676470588235 \\
\text { Água - NDVI }\end{array}$ & Voçoroca - declividade \\
\hline 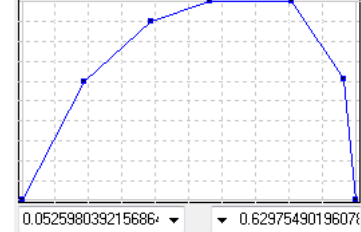 & 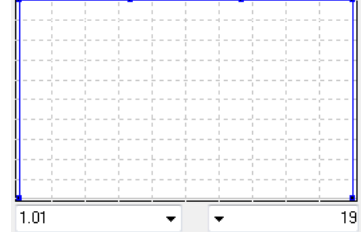 & 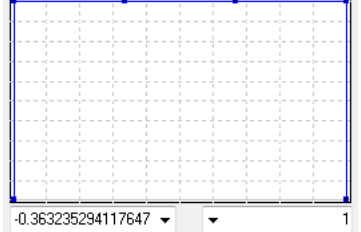 \\
\hline Vegetação - NDVI & Veg. arbórea - MDSN & Veg. rasteira - MDSN \\
\hline
\end{tabular}

\subsection{Classificação}

Neste estudo, o procedimento de classificação dos dados foi realizado pelos métodos de classificação hierárquica e árvores de decisão, com auxílio do programa 
eCognition Developer 8.8. A classificação hierárquica apresenta a vantagem de poder ser realizada usando diferentes níveis de segmentação, o que permite que os objetos sejam classificados no nível em que melhor são descritos por seu tamanho e forma. Entretanto, por permitir o uso do conhecimento do intérprete, necessita de um pré-conhecimento bastante específico sobre os alvos a serem classificados, demandando bastante tempo no estabelecimento da rede semântica e hierárquica, dos atributos e das regras de pertinência. Em virtude disso, a classificação foi realizada, comparativamente, com o algoritmo de árvore de decisão. O intuito foi verificar se a mineração dos dados, efetuada automaticamente por esse algoritmo, apresenta similaridade e coerência com o conjunto de regras estabelecido pelos autores. Os métodos e procedimentos são descritos na sequência.

\subsubsection{Classificação Baseada em Relações de Hierarquia}

Esse classificador foi aplicado aos objetos em diferentes níveis, provenientes da segmentação multirresolução. A figura 9a apresenta a rede semântica e a figura $9 \mathrm{~b}$ apresenta a rede hierárquica estabelecida em função dos níveis de segmentação com $\mathrm{FE}=150, \mathrm{FE}=30$ e $\mathrm{FE}=20$. No $\mathrm{FE}=150$ foi realizada a classificação das feições em vegetação e não vegetação, com menor nível de detalhamento dos segmentos. Para tal, foram usados os valores digitais da imagem NDVI. A partir dessa primeira classificação, no nível com FE 30, foram separados os objetos de vegetação em arbórea ou rasteira. Para tal, usaram-se as alturas dos objetos constantes no MDSN. Os objetos classificados como não vegetação foram reclassificados como água ou solo, em função dos valores digitais da banda do infravermelho próximo e da imagem NDVI. No nível com FE 20 foram reclassificados os objetos de solo, detalhando-os em solo exposto ou voçoroca. O atributo determinante nessa classificação foi a declividade dos objetos. A figura 9c apresenta os descritores e algoritmos classificadores (nearest neighbour e/ou fuzzy) utilizados para cada classe. A definição dos níveis de segmentação, dos dados de entrada, dos descritores e algoritmos classificadores foi realizada de forma empírica, e é apresentado neste estudo o conjunto selecionado que apresentou os melhores resultados de classificação.

\subsubsection{Classificação por Árvores de Decisão}

A classificação por árvores de decisão foi realizada com auxílio do algoritmo CART. Como dados de entrada foram usados: as quatro bandas da imagem IKONOS, a imagem NDVI, a imagem de intensidade, o MDSN, o MDS e o mapa de declividade. A classificação foi realizada sob os objetos originados pela segmentação com $\mathrm{FE}=50$, parâmetro de forma 0,1 e compacidade 0,5. Foram selecionadas amostras para as classes definidas na rede semântica. A partir das amostras, realizou-se o treinamento da árvore de decisão. Posteriormente, a classificação foi realizada pela árvore de decisão, em função das regras de decisão estabelecidas pela mineração dos dados. 
Figura 9 - Rede Semântica (a), Rede Hierárquica (b) e descritores das classes (c).

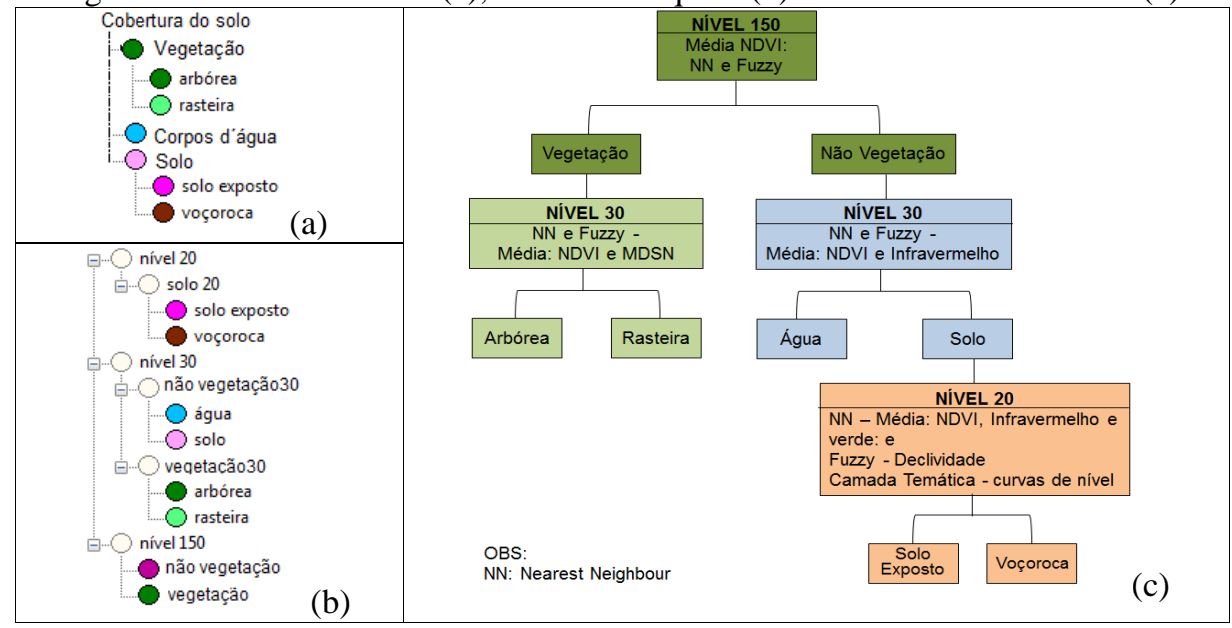

\section{RESULTADOS E DISCUSSÃO}

\subsection{Classificação Hierárquica}

A classificação hierárquica foi realizada para os objetos oriundos das segmentações realizadas com $\mathrm{FE}=150, \mathrm{FE}=30$ e $\mathrm{FE}=20$. Para os objetos do nível 150 foi realizada a classificação dos elementos de vegetação e não vegetação. Nesse nível, menos detalhado, não houve confusão entre as duas classes. Para o nível 30, os objetos "vegetação" foram reclassificados nas classes, arbórea e rasteira. Os objetos "não vegetação" foram divididos nas classes água e solo. Nesse nível, também não houve confusão entre as classes.

Os objetos classificados como solo no nível 30 foram reclassificados para o nível 20, sendo divididos nas classes, solo exposto e voçoroca. A tabela 1 mostra a matriz de erros obtida para os objetos selecionados como amostras de treinamento. Percebe-se que duas amostras selecionadas como solo exposto foram classificadas como voçoroca. A acurácia global nesse nível hierárquico foi de $95,12 \%$ e o índice Kappa $=0,90$.

Tabela 1 - Matriz de erros da classificação hierárquica dos objetos segmentados com $\mathrm{FE}=20$.

\begin{tabular}{c|c|c|c|c}
\hline Classe & Solo exposto & Voçoroca & SC & Acurácia Produtor (\%) \\
\hline Solo exposto & 25 & 2 & 27 & 92,6 \\
\hline Voçoroca & 0 & 14 & 14 & 100 \\
\hline SL & 25 & 16 & 41 & \multicolumn{1}{|c}{} \\
\cline { 1 - 4 } Acurácia Usuário (\%) & 100 & 87,5 & \multicolumn{2}{|c}{}
\end{tabular}

OBS: $\mathrm{SC}=$ soma parcial da coluna; $\mathrm{SL}=$ soma parcial da linha 
A figura 10 ilustra a classificação resultante. A declividade foi utilizada como atributo mais significativo na separação das classes solo exposto e voçoroca. Entretanto, a declividade no interior da cabeceira não é acentuada como é nas bordas. Devido a isso, ocorreram confusões no interior da voçoroca com a classe solo exposto. Entretanto, o atributo permitiu a delimitação do contorno da feição erosiva em estudo. Uma alternativa para melhorar este resultado é o uso de informações espaciais, a inserção de informações de contexto e atributos geométricos. Pode-se verificar também o erro de inclusão de elementos de vegetação arbórea na classe voçoroca, devido à declividade existente entre a copa das árvores e o solo nas bordas das áreas vegetadas (áreas circuladas em amarelo).

Figura 10 - Classificação hierárquica.

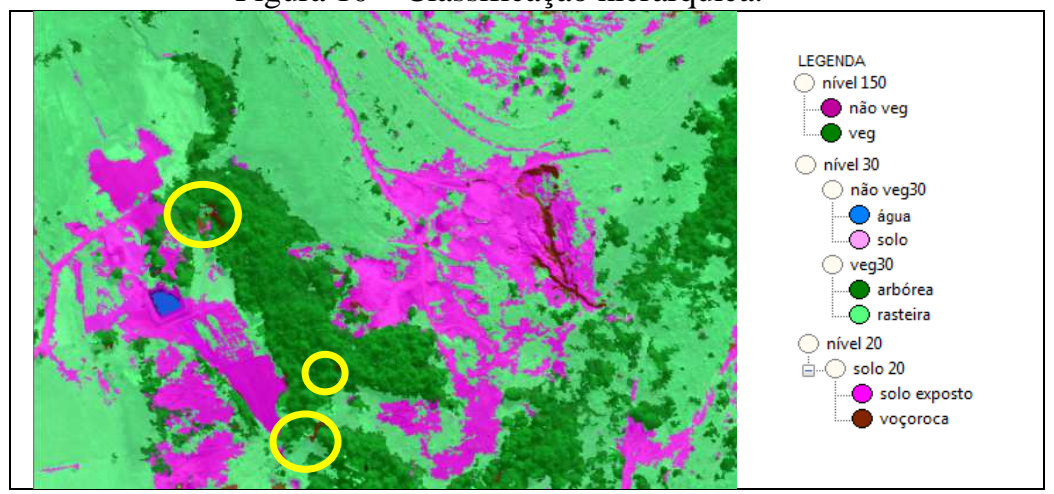

\subsection{Classificação por Árvore de Decisão}

A tabela 2 ilustra a matriz de erros da classificação realizada pelo classificador baseado em algoritmo de indução de árvore de decisão. Percebe-se que uma das amostras selecionadas como solo exposto foi classificada como voçoroca. A qualidade global foi de $98,8 \%$.

Tabela 2 - Matriz de erros da classificação por árvore de decisão.

\begin{tabular}{l|c|c|c|c|c|c|c}
\hline Classe & Água & Arbórea & Rasteira & $\begin{array}{c}\text { Solo } \\
\text { exposto }\end{array}$ & Voçoroca & \multicolumn{2}{|c}{$\begin{array}{c}\text { Acurácia } \\
\text { Produtor } \\
(\%)\end{array}$} \\
\hline Água & 3 & 0 & 0 & 0 & 0 & 3 & 100 \\
\hline Arbórea & 0 & 14 & 0 & 0 & 0 & 14 & 100 \\
\hline Rasteira & 0 & 0 & 14 & 0 & 0 & 14 & 100 \\
\hline Solo exposto & 0 & 0 & 0 & 30 & 1 & 31 & 96,8 \\
\hline Voçoroca & 0 & 0 & 0 & 0 & 18 & 18 & 100 \\
\hline SL & 3 & 14 & 14 & 30 & 19 & 80 & \\
\hline $\begin{array}{l}\text { Acurácia } \\
\text { Usuário (\%) }\end{array}$ & 100 & 100 & 100 & 100 & 94,7 & & \\
\cline { 1 - 5 } & & & & &
\end{tabular}

OBS: $\mathrm{SC}=$ soma parcial da coluna; $\mathrm{SL}=$ soma parcial da linha 
A figura 11 mostra a árvore de decisão gerada automaticamente pelo classificador em função das amostras de treinamento. Comparando-se com os atributos definidos para a classificação hierárquica (figura 10), percebe-se que a separação no primeiro ramo da árvore é similar à separação entre as classes vegetação e não vegetação, em função do NDVI. No ramo correspondente à vegetação, a separação entre as classes arbórea e rasteira foi realizada em função do MDSN, enquanto que na classificação hierárquica usou-se também o NDVI. No ramo correspondente à não vegetação, a separação entre as classes água e solo foi realizada em função apenas do NDVI, enquanto que na classificação hierárquica usou-se também a banda do infravermelho. Neste mesmo ramo, a separação entre as classes solo exposto e voçoroca se deu em função da declividade, enquanto que na classificação hierárquica foram usadas também as informações do NDVI e das bandas do verde e infravermelho. Na classificação hierárquica também foi usada, em algumas etapas da classificação, lógica fuzzy, diferenciando-se da classificação por árvore de decisão.

Analisando qualitativamente a classificação obtida por árvore de decisão (figura 12), e em relação ao resultado da classificação hierárquica (ambas baseadas em regiões), percebe-se um aumento das áreas classificadas como solo exposto em contraposição às de vegetação rasteira. Essa confusão entre as classes se deu nas regiões da cena em que as culturas aparecem em estágio inicial, nas quais é possível entrever o solo abaixo da vegetação. Não foram percebidas diferenças significativas entre as duas classificações em relação à classe vegetação arbórea. Para a classe voçoroca, as regiões internas da voçoroca foram classificadas corretamente, entretanto, para o entorno da mesma, houve um acréscimo na classificação, alterando o contorno da feição. Além disso, regiões de solo exposto foram incorporadas à classe.

Figura 11 - Árvore de decisão gerada com o algoritmo CART.

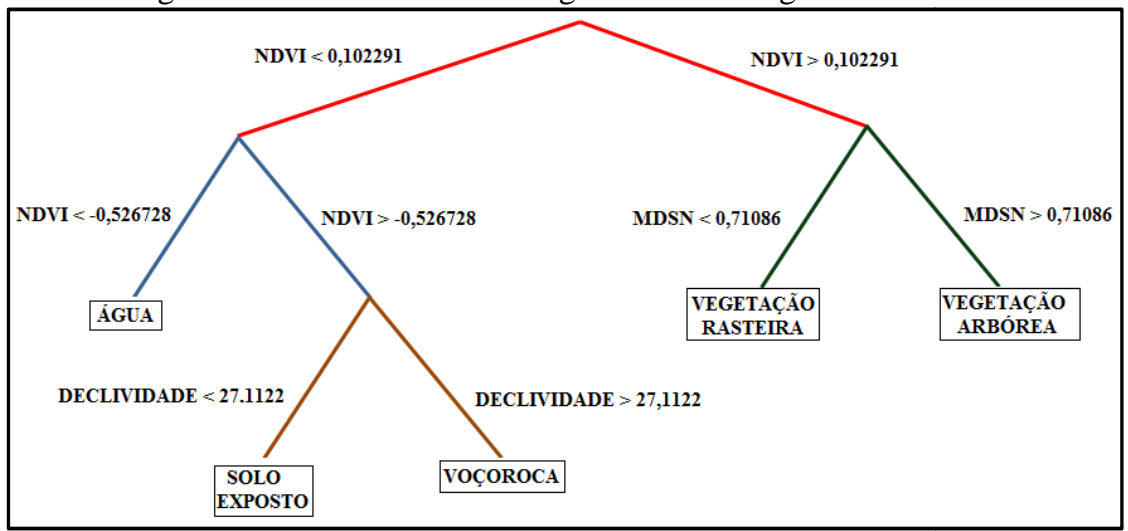

Bol. Ciênc. Geod., sec. Artigos, Curitiba, v. 20, no 4, p.1005-1026, out-dez, 2014. 
Figura 12 - Classificação por árvore de decisão.

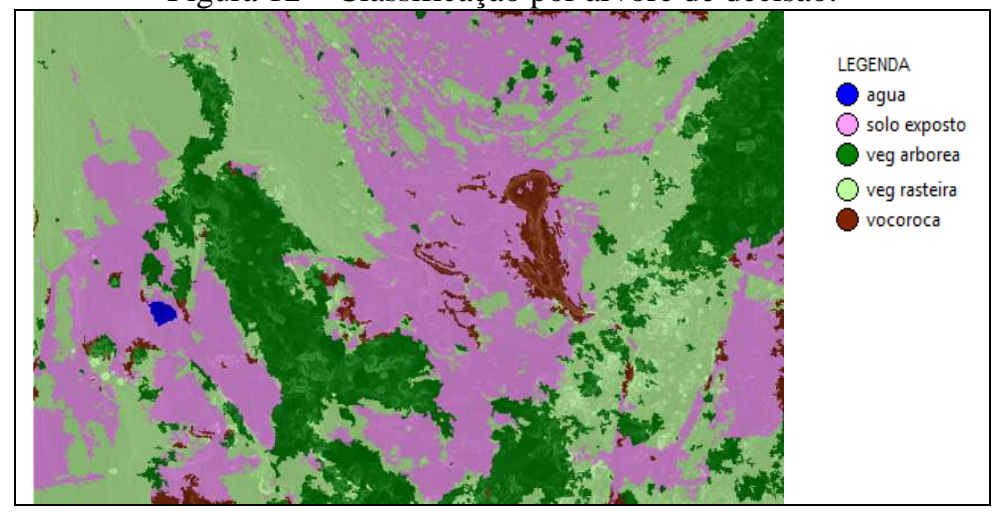

\section{CONCLUSÕES E RECOMENDAÇÕES}

Os resultados deste estudo comprovaram que a inserção de informações altimétricas auxilia na discriminação da voçoroca, mas não é suficiente para permitir a extração do contorno da voçoroca.

A classificação resultante do processamento baseado em objetos (regiões), e realizada de forma hierárquica, mostrou a separação entre as classes e um delineamento da feição voçoroca. Esse resultado comprova a eficácia do método proposto, bem como da rede hierárquica estabelecida e dos atributos selecionados para discriminação da feição de interesse. A classificação hierárquica foi desenvolvida com base no pré-conhecimento dos autores, requerendo maior tempo de análise para geração das segmentações, seleção de atributos e seus limiares, e para estabelecimento das regras de pertinência a cada classe. O uso do algoritmo CART agilizou consideravelmente o procedimento de classificação, e a árvore de decisão gerada automaticamente é muito semelhante às regras de decisão definidas para a classificação hierárquica. Considerando-se que a classificação resultante do algoritmo CART foi menos fidedigna à realidade do que a obtida de forma hierárquica, recomenda-se que a árvore de decisão seja gerada preliminarmente, de forma a conduzir a seleção de atributos e seus limiares, os quais devem posteriormente ser refinados de acordo com o conhecimento do intérprete.

Os resultados apresentados são preliminares e não foram usadas informações espaciais na obtenção dos mesmos. O refinamento da classificação hierárquica vem sendo conduzido e novos processamentos estão sendo realizados com a incorporação de dados geométricos e de contexto, os quais têm mostrado uma melhora significativa na extração do contorno da voçoroca. Para avaliar quantitativamente o resultado da classificação, será gerado um mapa de uso do solo, com base na interpretação visual sobre os objetos segmentados a ser usado usado como referência, e a matriz de erros será calculada em função do mesmo. A 
metodologia, após o refinamento e finalização da base de regras de decisão, será aplicada a uma área de verificação.

\section{AGRADECIMENTOS}

À Esteio Engenharia e Aerolevantamentos S.A., que cedeu os dados do perfilamento a laser aerotransportado.

À Engesat Imagens de Satélite, que cedeu a imagem do sensor IKONOS.

\section{REFERÊNCIAS}

ALKHASAWNEH, M.; NGAH, U. K.; TAY, L. T.; ISA, N. A. M.; AL-BATAH, M. S. Modeling and Testing Landslide Hazard Using Decision Tree. Journal of Applied Mathematics, volume 2014, article ID 929768. 2014. Accessed 11/08/2014. http://dx.doi.org/10.1155/2014/929768.

ANTUNES, A. F. B. Classificação de ambiente ciliar baseada em orientação a objeto em imagens de alta resolução espacial. 147 p. Tese (Doutorado em Ciências Geodésicas) - Setor de Ciências da Terra, Universidade Federal do Paraná, Curitiba-Pr. 2003.

AXELSSON, P. DEM generation from laserscanner data using adaptive TIN models. International Archives of Photogrammetry and Remote Sensing, XXXIII, Part B3.p. 85-92. 2000.

BAATZ, M.; SCHÄPE, A. Object-Oriented and Multi-Scale Image Analysis in Semantic Networks. Proc. of 2nd International Symposium: Operationalization of Remote Sensing, 16-20 August. Enschede ITC. 1999. Accessed 10/08/2014. http://www.ecognition.com/sites/default/files/409_itc 1999.pdf.

BREIMAN, L.; FRIEDMAN, J. H.; OLSEN, R. A.; STONE, C. J. Classification and Regression Trees. Belmont, CA: Wadsworth. 1984.

CENTENO, J. A. S. Sensoriamento remoto e processamento de imagens digitais.: Curso de Pós-Graduação em C. Geodésicas da UFPR. Curitiba - Pr. 2009.

DABA, S.; RIEGER, W.; STRAUSS, P. Assessment of gully erosion in eastern Ethiopia using photogrammetric techniques. Catena 50, p. 273-291. 2003.

EUSTACE, A.; PRINGLE, M.; WITTE, C. Give Me the Dirt: Detection of Gully Extent and Volume Using High-Resolution Lidar. In: JONES, S.; REINKE, K. Innovations in Remote Sensing and Photogrammetry. Springer. p. 255-269. 2009.

GONZALES, R. C.; WOODS, R. E. Digital image processing. $3^{\text {rd }}$ Edition. New York: Addison-Wesley Publishing Company Inc. 2008.

GUERRA, A. J. T. O início do processo erosivo. In: GUERRA, A. J. T. (Org.); SILVA, A. S.(Org.); BOTELHO, R. G. M. (Org.). Erosão e Conservação dos Solos: Conceitos, Temas e Aplicações. 2. ed. Rio de Janeiro: Bertran Brasil, p. 17-55. 2005.

HESSEL, R., VAN ASCH, T. Modelling gully erosion for a small catchment on the Chinese Loess Plateau. Catena 54, p.131-146. 2003. 
JAMES, L. A.; WATSON, D. G.; HANSEN, W. Using LiDAR data to map gullies and headwater streams under forest canopy: South Carolina, USA. Catena 71, p. 132-144. 2007.

JOHANSEN, K.; TAIHEI, S.; TINDALL, D.; PHINN, S. Object-based Monitoring of Gully Extent and Volume in North Australia using LiDAR Data. Proceedings of the 4th GEOBIA. May 7-9. Rio de Janeiro. p. 168-173. 2012.

KING, C.; BAGHDADI, N.; LECOMTE, V.; CERDAN, O. The application of remote-sensing data to monitoring and modelling of soil erosion. Catena 62, p. 79-93. 2005.

LANDIS, J. R.; KOCH, G. G.The measurement of observer agreement for category data. Biometrics, 33(1), p.159-174. 1977.

LEAL, P. C. B.; RODRIGUES, S. C. Uso e comparação do método de estaqueamento, o método DGPS e geoprocessamento no monitoramento de uma área erosiva na Fazenda do Glória - Uberlândia-MG. GEOUSP - Espaço e Tempo, São Paulo, no 29, p. 18-35. 2011.

MARTÍNEZ-CASASNOVAS, J. A. A spatial information technology approach for the mapping and quantification of gully erosion. Catena, volume 50, p. 293308. 2003.

MARTÍNEZ-CASASNOVAS, J. A.; RAMOS, M. C.; POESEN, J. Assessment of sidewall erosion in large gullies using multi-temporal DEMs and logistic regression analysis. Geomorphology 58, p. 305-321. 2004.

MARZOLFF, I; POESEN, J. The potential of 3D gully monitoring with GIS using high-resolution aerial photography and a digital photogrammetry system. Geomorphology 111, p. 48-60. 2009.

MORGAN, R. P. C. Soil Erosion and Conservation. Third Edition. Blackwell Publishing. 2005.

PERROY, R. L.; BOOKHAGEN, B.; ASNER, G. P.; CHADWICK, O. A. Comparison of gully erosion estimates using airborne and ground-based LiDAR on Santa Cruz Island, California. Geomorphology 118, p. 288-300. 2010.

SHRUTHI, R. B. V.; KERLE, N.; JETTEN, V. Object based gully feature extraction using high spatial resolution imagery. Geomorphology 134, p. 260268. 2011.

SHRUTHI, R. B. V.; KERLE, N.; JETTEN, V. Extracting gully features and its dynamics from high spatial resolution imagery using object based image analysis. Proceedings of the 4th GEOBIA. May 7-9. Rio de Janeiro-RJ. p. 7-12. 2012.

TRIMBLE. eCognition Developer 8.8 User Guide. Germany, 2012.

TSO, B.; MATHER, P. M. Classification Methods for Remotely Sensed Data. Second Edition. CRC Press. Florida. 2009.

VIEIRA, N. M. Estudo geomorfológico das voçorocas de Franca-SP. Tese Faculdade de Filosofia, Ciências e Letras da Universidade Estadual Paulista "Júlio de Mesquita Filho", Franca. 1978. 
VRIELING, A.; RODRIGUES, S. C.; STERK, G. Evaluating erosion from space: a case study near Uberlândia. Revista Sociedade \& Natureza, Special Issue, p. 683-696. Uberlândia - MG. 2005.

WANG, T.; HE, F.; ZHANG, A.; GU, L.; WEN, Y.; JIANG, W.; SHAO, H. A Quantitative Study of Gully Erosion Based on Object-Oriented Analysis Techniques: A Case Study in Beiyanzikou Catchment of Qixia, Shandong, China. The Scientific World Journal, volume 2014, article ID 417325. 2014.

ZHANG, K.; CUI, Z. Airborne LIDAR Data Processing and Analysis Tools. National Center for Airborne Laser Mapping. 2007.

(Recebido em maio de 2014. Aceito em setembro de 2014). 